\title{
Approximation of Common Fixed Points of a Sequence of Nearly Nonexpansive Mappings and Solutions of Variational Inequality Problems
}

\author{
D. R. Sahu, ${ }^{1}$ Shin Min Kang, ${ }^{2}$ and Vidya Sagar ${ }^{1}$ \\ ${ }^{1}$ Department of Mathematics, Banaras Hindu University, Varanasi 221005, India \\ ${ }^{2}$ Department of Mathematics and RINS, Gyeongsang National University, Jinju 660-701, Republic of Korea \\ Correspondence should be addressed to Shin Min Kang, smkang@gnu.ac.kr
}

Received 10 April 2012; Accepted 14 May 2012

Academic Editor: Yonghong Yao

Copyright (c) 2012 D. R. Sahu et al. This is an open access article distributed under the Creative Commons Attribution License, which permits unrestricted use, distribution, and reproduction in any medium, provided the original work is properly cited.

We introduce an explicit iterative scheme for computing a common fixed point of a sequence of nearly nonexpansive mappings defined on a closed convex subset of a real Hilbert space which is also a solution of a variational inequality problem. We prove a strong convergence theorem for a sequence generated by the considered iterative scheme under suitable conditions. Our strong convergence theorem extends and improves several corresponding results in the context of nearly nonexpansive mappings.

\section{Introduction}

Let $C$ be a nonempty subset of a real Hilbert space $H$ with inner product $\langle\cdot, \cdot\rangle$ and norm $\|\cdot\|$, respectively. A mapping $T: C \rightarrow H$ is called the following:

(1) monotone if

$$
\langle T x-T y, x-y\rangle \geq 0 \quad \forall x, y \in C,
$$

(2) $\eta$-strongly monotone if there exists a positive real number $\eta$ such that

$$
\langle T x-T y, x-y\rangle \geq \eta\|x-y\|^{2} \quad \forall x, y \in C,
$$

(3) $k$-Lipschitzian if there exists a constant $k>0$ such that

$$
\|T x-T y\| \leq k\|x-y\| \quad \forall x, y \in C,
$$


(4) nonexpansive if

$$
\|T x-T y\| \leq\|x-y\| \quad \forall x, y \in C
$$

(5) nearly nonexpansive $[1,2]$ with respect to a fixed sequence $\left\{a_{\mathrm{n}}\right\}$ in $[0, \infty)$ with $a_{n} \rightarrow 0$ if

$$
\left\|T^{n} x-T^{n} y\right\| \leq\|x-y\|+a_{n} \quad \forall x, y \in C \text { and } n \in \mathbb{N} .
$$

In [3], Moudafi proposed viscosity approximation methods of selecting a particular fixed point of a given nonexpansive mapping in Hilbert spaces (see [4] for further developments in both Hilbert and Banach spaces). Let $f$ be a contraction on $H$. Starting with an arbitrary initial $x_{1} \in H$, define a sequence $\left\{x_{n}\right\}$ recursively by

$$
x_{n+1}=\alpha_{n} f\left(x_{n}\right)+\left(1-\alpha_{n}\right) T x_{n} \quad \forall n \in \mathbb{N},
$$

where $\left\{\alpha_{n}\right\}$ is a sequence in $(0,1)$. It is proved in [4] that under appropriate conditions imposed on $\left\{\alpha_{n}\right\}$, the sequence $\left\{x_{n}\right\}$ generated by (1.6) strongly converges to the unique solution $x^{*} \in C$ of the variational inequality

$$
\left\langle(I-f) x^{*}, x-x^{*}\right\rangle \geq 0 \quad \forall x \in C,
$$

where $C=F(T)$, the set of fixed points of $T$.

In 2006, Marino and Xu [5] introduced the viscosity iterative method for nonexpansive mappings. Starting with an arbitrary initial $x_{1} \in H$, define a sequence $\left\{x_{n}\right\}$ recursively by

$$
x_{n+1}=\alpha_{n} \gamma f\left(x_{n}\right)+\left(I-\alpha_{n} A\right) T x_{n} \quad \forall n \in \mathbb{N} .
$$

They proved that the sequence $\left\{x_{n}\right\}$ generated by (1.8) converges strongly to the unique solution of the variational inequality

$$
\left\langle(A-\gamma f) x^{*}, x-x^{*}\right\rangle \geq 0 \quad \forall x \in C
$$

which is the optimality condition for the minimization problem

$$
\min _{x \in C} \frac{1}{2}\langle A x, x\rangle-h(x)
$$

where $h$ is a potential function for $\gamma f$ (i.e., $h^{\prime}(x)=\gamma f(x)$ for all $x \in H$ ), and $A$ is a strongly positive bounded linear operator on $H$; that is, there is a constant $\bar{\gamma}>0$ such that

$$
\langle A x, x\rangle \geq \bar{\gamma}\|x\|^{2} \quad \forall x \in H .
$$


The applications of the iterative method (1.8) have been studied by some researchers (see $[6,7])$.

Also, Wang [8,9] and Wang and Hu [10] introduced the iterative method for nonexpansive mappings.

Recently, Tian [11] proposed an implicit and an explicit schemes on combining the iterative methods of Marino and $\mathrm{Xu}$ [5] and Yamada [12]. He also proved the strong convergence of these two schemes to a fixed point of a nonexpansive mapping $T$ defined on a real Hilbert space under suitable conditions.

More recently, Ceng et al. [13] introduced an implicit and an explicit schemes using the properties of projection for finding the fixed points of a nonexpansive mapping defined on the closed convex subset of a real Hilbert space. They also proved the strong convergence of the sequences generated by the proposed schemes to a fixed point of a nonexpansive mapping which is also a solution of a variational inequality defined on the set of fixed points.

Aoyama et al. [14] proved strong convergence of an iterative scheme for a sequence of nonexpansive mappings as follows.

Theorem 1.1. Let $X$ be a uniformly convex Banach space whose norm is uniformly Gateaux differentiable and $C$ be a nonempty closed convex subset of $X$. Let $\left\{T_{n}\right\}$ be a sequence of nonexpansive mappings from $C$ into itself such that $\bigcap_{n=1}^{\infty} F\left(T_{n}\right) \neq \emptyset$. Let $T$ be a mapping from $C$ into itself defined by $T x=\lim _{n \rightarrow \infty} T_{n} x$ for all $x \in C$ and $F(T)=\bigcap_{n=1}^{\infty} F\left(T_{n}\right)$. Let $\left\{x_{n}\right\}$ be a sequence in $C$ generated by the following iterative process:

$$
\begin{gathered}
x_{1}=x \in C, \\
x_{n+1}=\alpha_{n} x+\left(1-\alpha_{n}\right) T_{n} x_{n} \quad \forall n \in \mathbb{N},
\end{gathered}
$$

where $\left\{\alpha_{n}\right\}$ is a sequence in $[0,1]$ satisfying the following conditions:

(a) $\lim _{n \rightarrow \infty} \alpha_{n}=0$ and $\sum_{n=1}^{\infty} \alpha_{n}=\infty$;

(b) either $\sum_{n=1}^{\infty}\left|\alpha_{n+1}-\alpha_{n}\right|<\infty$ or $\alpha_{n} \in(0,1]$ and $\lim _{n \rightarrow \infty} \alpha_{n+1} / \alpha_{n}=1$;

(c) $\sum_{n=1}^{\infty} \sup \left\{\left\|T_{n} z-T_{n+1} z\right\|: z \in B\right\}<\infty$ for any bounded subset $B$ of $C$.

Then, the sequence $\left\{x_{n}\right\}$ converges strongly to $Q x$, where $Q$ is the sunny nonexpansive retraction from $X$ onto $F(T)$.

Let $C$ be a nonempty subset of a real Hilbert space $H$. Let $\tau:=\left\{T_{n}\right\}$ be a sequence of mappings from $C$ into itself. We denote by $F(\tau)$ the set of common fixed points of the sequence $\tau$, that is, $F(\tau)=\bigcap_{n=1}^{\infty} F\left(T_{n}\right)$. Fix a sequence $\left\{a_{n}\right\}$ in $[0, \infty)$ with $a_{n} \rightarrow 0$, and let $\left\{T_{n}\right\}$ be a sequence of mappings from $C$ into $H$. Then, the sequence $\left\{T_{n}\right\}$ is called a sequence of nearly nonexpansive mappings [15] with respect to a sequence $\left\{a_{n}\right\}$ if

$$
\left\|T_{n} x-T_{n} y\right\| \leq\|x-y\|+a_{n} \quad \forall x, y \in C, n \in \mathbb{N} .
$$

It is obvious that the sequence of nearly nonexpansive mappings is a wider class of sequence of nonexpansive mappings.

In this paper, inspired by Aoyama et al. [14], Ceng et al. [13], and Sahu et al. [15], we introduce an explicit iterative scheme and prove a strong convergence theorem for computing an element of $F(\tau)$, the set of common fixed points of a sequence $\tau=\left\{T_{n}\right\}$ of nearly 
nonexpansive mappings which is also a solution of a variational inequality over $F(\tau)$. Our result generalizes and improves the results of Ceng et al. [13], Tian [11], and many other related works.

\section{Preliminaries}

Throughout this paper, we denote by $I$ the identity operator of $H$. Also, we denote by $\rightarrow$ and $\rightarrow$ the strong convergence and weak convergence, respectively. The symbol $\mathbb{N}$ stands for the set of all natural numbers.

Let $C$ be a nonempty closed convex subset of a real Hilbert space $H$. Then, for any $x \in H$, there exists a unique nearest point in $C$, denoted by $P_{C}(x)$, such that

$$
\left\|x-P_{C}(x)\right\| \leq\|x-y\| \quad \forall y \in C .
$$

The mapping $P_{C}$ is called the metric projection from $H$ onto $C$ (see [1]).

Let $C$ be a nonempty subset of a real Hilbert space $H$ and $T_{1}, T_{2}: C \rightarrow H$ be two mappings. We denote $B(C)$, the collection of all bounded subsets of $C$. The deviation between $T_{1}$ and $T_{2}$ on $B \in B(C)$, denoted by $\Phi_{B}\left(T_{1}, T_{2}\right)$, is defined by

$$
\Phi_{B}\left(T_{1}, T_{2}\right)=\sup \left\{\left\|T_{1} x-T_{2} x\right\|: x \in B\right\} .
$$

The following lemmas will be needed to prove our main result.

Lemma 2.1 (see [16]). The metric projection mapping $P_{C}$ is characterized by the following properties:

(a) $P_{C}(x) \in C$ for all $x \in H$;

(b) $\left\langle x-P_{C}(x), P_{C}(x)-y\right\rangle \geq 0$ for all $x \in H$ and $y \in C$;

(c) $\|x-y\|^{2} \geq\left\|x-P_{C}(x)\right\|^{2}+\left\|y-P_{C}(x)\right\|^{2}$ for all $x \in H$ and $y \in C$;

(d) $\left\langle P_{C}(x)-P_{C}(y), x-y\right\rangle \geq\left\|P_{C}(x)-P_{C}(y)\right\|^{2}$ for all $x, y \in H$.

Lemma 2.2 (see [13]). Let $V: C \rightarrow H$ be an L-Lipschitzian mapping and $F: C \rightarrow H$ be a $k$ Lipschitzian and $\eta$-strongly monotone operator. Then, for $0 \leq \gamma L<\mu \eta$,

$$
\langle x-y,(\mu F-\gamma V) x-(\mu F-\gamma V) y\rangle \geq(\mu \eta-\gamma L)\|x-y\|^{2} \quad \forall x, y \in C .
$$

That is, $\mu F-\gamma V$ is strongly monotone with coefficient $\mu \eta-\gamma L$.

Lemma 2.3 (see [12]). Let $C$ be a nonempty subset of a real Hilbert space $H$. Suppose that $\lambda \in(0,1)$ and $\mu>0$. Let $F: C \rightarrow H$ be a $k$-Lipschitzian and $\eta$-strongly monotone operator on $C$. Define the mapping $\mathrm{G}: \mathrm{C} \rightarrow \mathrm{H}$ by

$$
G x=x-\lambda \mu F x \quad \forall x \in C
$$

Then $G$ is a contraction that provided $\mu<2 \eta / k^{2}$. More precisely, for $\mu \in\left(0,2 \eta / k^{2}\right)$,

$$
\|G x-G y\| \leq(1-\lambda \tau)\|x-y\| \quad \forall x, y \in C,
$$

where $\tau=1-\sqrt{1-\mu\left(2 \eta-\mu k^{2}\right)} \in(0,1]$. 
Lemma 2.4 (see [1]). Let $C$ be a nonempty closed convex subset of a real Hilbert space $H$. Let $T: C \rightarrow C$ be a nonexpansive mapping. Then $I-T$ is demiclosed at zero; that is, if $\left\{x_{n}\right\}$ is a sequence in $C$ weakly converging to some $x \in C$ and the sequence $\left\{(I-T) x_{n}\right\}$ converges strongly to 0 , then $x \in F(T)$.

Lemma 2.5 (see [17]). Assume that $\left\{t_{n}\right\}$ is a sequence of nonnegative real numbers such that

$$
t_{n+1} \leq\left(1-\alpha_{n}\right) t_{n}+\alpha_{n} \beta_{n} \quad \forall n \in \mathbb{N},
$$

where $\left\{\alpha_{n}\right\}$ and $\left\{\beta_{n}\right\}$ are sequences of nonnegative real numbers which satisfy the following conditions:

(a) $\left\{\alpha_{n}\right\}_{n=1}^{\infty} \subset(0,1)$ and $\sum_{n=1}^{\infty} \alpha_{n}=\infty$;

(b) $\limsup _{n \rightarrow \infty} \beta_{n} \leq 0$, or

(b') $\sum_{n=1}^{\infty} \alpha_{n} \beta_{n}$ is convergent.

Then $\lim _{n \rightarrow \infty} t_{n}=0$.

Lemma 2.6 (see [18]). Let $C$ be a nonempty closed convex subset of a real Hilbert space $H$ and $\lambda_{i}>0 .(i=1,2,3, \ldots, N)$ such that $\sum_{i=1}^{N} \lambda_{i}=1$. Let $T_{1}, T_{2}, T_{3}, \ldots, T_{N}: C \rightarrow C$ be nonexpansive mappings such that $\bigcap_{i=1}^{N} F\left(T_{i}\right) \neq \emptyset$, and let $T=\sum_{i=1}^{N} \lambda_{i} T_{i}$. Then $T$ is nonexpansive from $C$ into itself and $F(T)=\bigcap_{i=1}^{N} F\left(T_{i}\right)$.

\section{Main Result}

Theorem 3.1. Let $C$ be a nonempty closed convex subset of a real Hilbert space $H$. Let $F: C \rightarrow H$ be a $k$-Lipschitzian and $\eta$-strongly monotone operator and $V: C \rightarrow H$ be an L-Lipschitzian mapping. Let $\tau=\left\{T_{n}\right\}$ be a sequence of nearly nonexpansive mappings from $C$ into itself with respect to a sequence $\left\{a_{n}\right\}$ such that $F(\tau) \neq \emptyset$ and $T$ be a mapping from $C$ into itself defined by $T x=\lim _{n \rightarrow \infty} T_{n} x$ for all $x \in C$. Suppose that $F(T)=F(\tau), 0<\mu<2 \eta / k^{2}$ and $0 \leq \gamma L<\tau$, where

$\tau=1-\sqrt{1-\mu\left(2 \eta-\mu k^{2}\right)}$. For an arbitrary $x_{1} \in C$, consider the sequence $\left\{x_{n}\right\}$ in $C$ generated by the following iterative process:

$$
\begin{gathered}
x_{1} \in C, \\
x_{n+1}=P_{C}\left[\alpha_{n} \gamma V x_{n}+\left(I-\alpha_{n} \mu F\right) T_{n} x_{n}\right] \quad \forall n \in \mathbb{N},
\end{gathered}
$$

where $\left\{\alpha_{n}\right\}$ is a sequence in $(0,1)$ satisfying the conditions:

(a) $\lim _{n \rightarrow \infty} \alpha_{n}=0$ and $\sum_{n=1}^{\infty} \alpha_{n}=\infty$;

(b) either $\sum_{n=1}^{\infty}\left|\alpha_{n+1}-\alpha_{n}\right|<\infty$ or $\lim _{n \rightarrow \infty} \alpha_{n+1} / \alpha_{n}=1$;

(c) either $\sum_{n=1}^{\infty} \Phi_{B}\left(T_{n}, T_{n+1}\right)<\infty$ or $\lim _{n \rightarrow \infty} \Phi_{B}\left(T_{n}, T_{n+1}\right) / \alpha_{n+1}=0$ for each $B \in B(C)$;

(d) $\lim _{n \rightarrow \infty} a_{n} / \alpha_{n}=0$.

Then, the sequence $\left\{x_{n}\right\}$ converges strongly to $\tilde{x} \in F(\tau)$, where $\tilde{x}$ is the unique solution of variational inequality

$$
\langle(\mu F-\gamma V) \tilde{x}, \tilde{x}-y\rangle \leq 0 \quad \forall y \in F(\tau)
$$


Proof. Let $T$ be a mapping from $C$ into itself defined by $T x=\lim _{n \rightarrow \infty} T_{n} x$ for all $x \in C$. It is clear that $T$ is a nonexpansive mapping. So, we have $F(T) \neq \emptyset$. Now, we proceed with the following steps.

Step 1. ( $\left\{x_{n}\right\}$ is bounded). Let $z \in F(\tau)$. From (3.1), we have

$$
\begin{aligned}
\left\|x_{n+1}-z\right\| & =\left\|P_{C}\left[\alpha_{n} \gamma V x_{n}+\left(I-\alpha_{n} \mu F\right) T_{n} x_{n}\right]-P_{C}(z)\right\| \\
& \leq\left\|\alpha_{n} \gamma V x_{n}+\left(I-\alpha_{n} \mu F\right) T_{n} x_{n}-z\right\| \\
& \leq\left\|\alpha_{n}\left(\gamma V x_{n}-\mu F z\right)+\left(I-\alpha_{n} \mu F\right) T_{n} x_{n}-\left(I-\alpha_{n} \mu F\right) T_{n} z\right\| \\
& \leq \alpha_{n} \gamma L\left\|x_{n}-z\right\|+\alpha_{n}\|(\gamma V-\mu F) z\|+\left(1-\alpha_{n} \tau\right)\left(\left\|x_{n}-z\right\|+a_{n}\right) \\
& \leq\left(1-\alpha_{n}(\tau-\gamma L)\right)\left\|x_{n}-z\right\|+\alpha_{n}\|(\gamma V-\mu F) z\|+\left(1-\alpha_{n} \tau\right) a_{n} \\
& \leq\left(1-\alpha_{n}(\tau-\gamma L)\right)\left\|x_{n}-z\right\|+\alpha_{n}\|(\gamma V-\mu F) z\|+a_{n} .
\end{aligned}
$$

Note that $\lim _{n \rightarrow \infty} a_{n} / \alpha_{n}=0$, so there exists a constant $K>0$ such that

$$
\frac{\alpha_{n}\|(\gamma V-\mu F) z\|+a_{n}}{\alpha_{n}} \leq K \quad \forall n \in \mathbb{N}
$$

Thus, we have

$$
\begin{aligned}
\left\|x_{n+1}-z\right\| & \leq\left(1-\alpha_{n}(\tau-\gamma L)\right)\left\|x_{n}-z\right\|+\alpha_{n} K \\
& \leq \max \left\{\left\|x_{n}-z\right\|, \frac{K}{\tau-\gamma L}\right\} \quad \forall n \in \mathbb{N} .
\end{aligned}
$$

Hence, $\left\{x_{n}\right\}$ is bounded. So $\left\{T_{n} x_{n}\right\}$ and $\left\{V x_{n}\right\}$ are bounded.

Step 2. $\left(\left\|x_{n+1}-x_{n}\right\| \rightarrow 0\right.$ as $\left.n \rightarrow \infty\right)$. From (3.1), we have

$$
\begin{aligned}
\left\|x_{n+1}-x_{n}\right\|= & \left\|P_{C}\left[\alpha_{n} \gamma V x_{n}+\left(I-\alpha_{n} \mu F\right) T_{n} x_{n}\right]-P_{C}\left[\alpha_{n-1} \gamma V x_{n-1}+\left(I-\alpha_{n-1} \mu F\right) T_{n-1} x_{n-1}\right]\right\| \\
\leq & \left\|\left[\alpha_{n} \gamma V x_{n}+\left(I-\alpha_{n} \mu F\right) T_{n} x_{n}\right]-\left[\alpha_{n-1} \gamma V x_{n-1}+\left(I-\alpha_{n-1} \mu F\right) T_{n-1} x_{n-1}\right]\right\| \\
\leq & \| \alpha_{n} \gamma\left(V x_{n}-V x_{n-1}\right)+\gamma\left(\alpha_{n}-\alpha_{n-1}\right) V x_{n-1} \\
& +\left(I-\alpha_{n} \mu F\right) T_{n} x_{n}-\left(I-\alpha_{n} \mu F\right) T_{n} x_{n-1} \\
& +T_{n} x_{n-1}-T_{n-1} x_{n-1}+\alpha_{n-1} \mu F T_{n-1} x_{n-1}-\alpha_{n} \mu F T_{n} x_{n-1} \| \\
\leq & \alpha_{n} \gamma L\left\|x_{n}-x_{n-1}\right\|+\left\|\gamma\left(\alpha_{n}-\alpha_{n-1}\right) V x_{n-1}\right\| \\
& +\left(1-\alpha_{n} \tau\right)\left\|T_{n} x_{n}-T_{n} x_{n-1}\right\|+\left\|T_{n} x_{n-1}-T_{n-1} x_{n-1}\right\| \\
& +\mu\left\|\alpha_{n-1} F T_{n-1} x_{n-1}-\alpha_{n} F T_{n} x_{n-1}\right\|
\end{aligned}
$$


Journal of Applied Mathematics

$$
\begin{aligned}
\leq & \left(1-\alpha_{n}(\tau-\gamma L)\right)\left\|x_{n}-x_{n-1}\right\|+\Phi_{B}\left(T_{n}, T_{n-1}\right)+\left(1-\alpha_{n} \tau\right) a_{n} \\
& +\left\|\gamma\left(\alpha_{n}-\alpha_{n-1}\right) V x_{n-1}\right\| \\
& +\mu\left\|\alpha_{n-1}\left(F T_{n-1} x_{n-1}-F T_{n} x_{n-1}\right)-\left(\alpha_{n}-\alpha_{n-1}\right)\left(F T_{n} x_{n-1}\right)\right\| \\
\leq & \left(1-\alpha_{n}(\tau-\gamma L)\right)\left\|x_{n}-x_{n-1}\right\|+\Phi_{B}\left(T_{n}, T_{n-1}\right)\left(1+\mu \alpha_{n-1} k\right) \\
& +M\left|\alpha_{n}-\alpha_{n-1}\right|+a_{n},
\end{aligned}
$$

for some constant $M>0$. Thus, using Lemma 2.5, we get $\left\|x_{n+1}-x_{n}\right\| \rightarrow 0$ as $n \rightarrow \infty$.

Step 3. We have $\left(\left\|x_{n}-T x_{n}\right\| \rightarrow 0\right.$ as $\left.n \rightarrow \infty\right)$. Note that

$$
\begin{aligned}
\left\|x_{n}-T_{n} x_{n}\right\| & \leq\left\|x_{n}-x_{n+1}\right\|+\left\|x_{n+1}-T_{n} x_{n}\right\| \\
& =\left\|x_{n}-x_{n+1}\right\|+\left\|P_{C}\left[\alpha_{n} \gamma V x_{n}+\left(I-\alpha_{n} \mu F\right) T_{n} x_{n}\right]-P_{C}\left(T_{n} x_{n}\right)\right\| \\
& \leq\left\|x_{n}-x_{n+1}\right\|+\left\|\alpha_{n} \gamma V x_{n}+\left(I-\alpha_{n} \mu F\right) T_{n} x_{n}-T_{n} x_{n}\right\| \\
& =\left\|x_{n}-x_{n+1}\right\|+\alpha_{n}\left\|\gamma V x_{n}-\mu F T_{n} x_{n}\right\| \longrightarrow 0 \quad \text { as } n \longrightarrow \infty .
\end{aligned}
$$

Since

$$
\begin{aligned}
\left\|x_{n}-T x_{n}\right\| & \leq\left\|x_{n}-T_{n} x_{n}\right\|+\left\|T_{n} x_{n}-T x_{n}\right\| \\
& \leq\left\|x_{n}-T_{n} x_{n}\right\|+\Phi_{B}\left(T_{n}, T\right),
\end{aligned}
$$

it follows that $\lim _{n \rightarrow \infty}\left\|x_{n}-T x_{n}\right\|=0$.

Step 4 . We have $\left(\lim \sup _{n \rightarrow \infty}\left\langle x_{n}-\tilde{x},(\gamma V-\mu F) \tilde{x}\right\rangle \leq 0\right)$. Let us choose a subsequence $\left\{x_{n_{k}}\right\}$ of $\left\{x_{n}\right\}$ such that

$$
\limsup _{n \rightarrow \infty}\left\langle x_{n}-\tilde{x},(\gamma V-\mu F) \tilde{x}\right\rangle=\lim _{k \rightarrow \infty}\left\langle x_{n_{k}}-\tilde{x},(\gamma V-\mu F) \tilde{x}\right\rangle .
$$

Without loss of generality, we may assume that $x_{n_{k}} \rightarrow z \in C$. By using Lemma 2.4, we get that $z \in F(T)$. Note that $F(T)=F(\tau)$, it follows that $z \in F(\tau)$. Hence from (3.2), we get the following:

$$
\limsup _{n \rightarrow \infty}\left\langle x_{n}-\tilde{x},(\gamma V-\mu F) \tilde{x}\right\rangle=\langle z-\tilde{x},(\gamma V-\mu F) \tilde{x}\rangle \leq 0
$$

Step 5. We have $\left(x_{n} \rightarrow \tilde{x}\right.$ as $\left.n \rightarrow \infty\right)$. Set $y_{n}=\alpha_{n} \gamma V x_{n}+\left(I-\alpha_{n} \mu F\right) T_{n} x_{n}$ and $\gamma_{n}=$ $\alpha_{n}(\tau-\gamma L)$. Noticing that $x_{n+1}=P_{C}\left(y_{n}\right)$. From (3.1), we have

$$
\begin{aligned}
\left\|x_{n+1}-\tilde{x}\right\|^{2} & =\left\langle y_{n}-\tilde{x}, x_{n+1}-\tilde{x}\right\rangle+\left\langle P_{C}\left(y_{n}\right)-y_{n}, P_{C}\left(y_{n}\right)-\tilde{x}\right\rangle \\
& \leq\left\langle y_{n}-\tilde{x}, x_{n+1}-\tilde{x}\right\rangle
\end{aligned}
$$




$$
\begin{aligned}
= & \alpha_{n}\left\langle\gamma V x_{n}-\mu F \tilde{x}, x_{n+1}-\tilde{x}\right\rangle \\
& +\left\langle\left(I-\alpha_{n} \mu F\right) T_{n} x_{n}-\left(I-\alpha_{n} \mu F\right) T_{n} \tilde{x}, x_{n+1}-\tilde{x}\right\rangle \\
= & \alpha_{n} \gamma\left\langle V x_{n}-V \tilde{x}, x_{n+1}-\tilde{x}\right\rangle+\alpha_{n}\left\langle\gamma V \tilde{x}-\mu F \tilde{x}, x_{n+1}-\tilde{x}\right\rangle \\
& +\left\langle\left(I-\alpha_{n} \mu F\right) T_{n} x_{n}-\left(I-\alpha_{n} \mu F\right) T_{n} \tilde{x}, x_{n+1}-\tilde{x}\right\rangle \\
\leq & \alpha_{n} \gamma L\left\|x_{n}-\tilde{x}\right\|\left\|x_{n+1}-\tilde{x}\right\|+\alpha_{n}\left\langle(\gamma V-\mu F) \tilde{x}, x_{n+1}-\tilde{x}\right\rangle \\
& +\left(1-\alpha_{n} \tau\right)\left(\left\|x_{n}-\tilde{x}\right\|+a_{n}\right)\left\|x_{n+1}-\tilde{x}\right\| \\
= & \left(1-\alpha_{n}(\tau-\gamma L)\right)\left\|x_{n}-\tilde{x}\right\|\left\|x_{n+1}-\tilde{x}\right\| \\
& +\alpha_{n}\left\langle(\gamma V-\mu F) \tilde{x}, x_{n+1}-\tilde{x}\right\rangle+\left(1-\alpha_{n} \tau\right) a_{n}\left\|x_{n+1}-\tilde{x}\right\| \\
\leq & \left(1-\alpha_{n}(\tau-\gamma L)\right) \frac{1}{2}\left(\left\|x_{n}-\tilde{x}\right\|^{2}+\left\|x_{n+1}-\tilde{x}\right\|^{2}\right) \\
& +\alpha_{n}\left\langle(\gamma V-\mu F) \tilde{x}, x_{n+1}-\tilde{x}\right\rangle+a_{n}\left\|x_{n+1}-\tilde{x}\right\| .
\end{aligned}
$$

Hence, we have

$$
\begin{aligned}
\left\|x_{n+1}-\tilde{x}\right\|^{2} \leq & \frac{1-\alpha_{n}(\tau-\gamma L)}{1+\alpha_{n}(\tau-\gamma L)}\left\|x_{n}-\tilde{x}\right\|^{2}+\frac{2 \alpha_{n}}{1+\gamma_{n}}\left\langle(\gamma V-\mu F) \tilde{x}, x_{n+1}-\tilde{x}\right\rangle \\
& +\frac{2 a_{n}}{1+\gamma_{n}}\left\|x_{n+1}-\tilde{x}\right\| \\
\leq & \left(1-\alpha_{n}(\tau-\gamma L)\right)\left\|x_{n}-\tilde{x}\right\|^{2}+\frac{2 \alpha_{n}}{1+\gamma_{n}}\left\langle(\gamma V-\mu F) \tilde{x}, x_{n+1}-\tilde{x}\right\rangle \\
& +\frac{2 a_{n}}{1+\gamma_{n}}\left\|x_{n+1}-\tilde{x}\right\| \\
= & \left(1-\gamma_{n}\right)\left\|x_{n}-\tilde{x}\right\|^{2}+\gamma_{n} \delta_{n}+\frac{2 a_{n}}{1+\gamma_{n}}\left\|x_{n+1}-\tilde{x}\right\|
\end{aligned}
$$

where

$$
\delta_{n}=\frac{2}{\left(1+\gamma_{n}\right)(\tau-\gamma L)}\left\langle(\gamma V-\mu F) \tilde{x}, x_{n+1}-\tilde{x}\right\rangle
$$

Noticing that $\lim _{n \rightarrow \infty} a_{n} / \alpha_{n}=0$, it follows from Lemma 2.5 that $\lim _{n \rightarrow \infty} x_{n}=\tilde{x}$. This completes the proof. corollary.

Now, we derive the main result of Ceng et al. ([13], Theorem 3.2) as the following

Corollary 3.2. Let $C$ be a nonempty closed convex subset of a real Hilbert space $H$. Let $F: C \rightarrow H$ be a $k$-Lipschitzian and $\eta$-strongly monotone operator and $V: C \rightarrow H$ be an L-Lipschitzian mapping. Let $T: C \rightarrow C$ be a nonexpansive mapping such that $F(T) \neq \emptyset$. Suppose that $0<\mu<2 \eta / k^{2}$ and 
$0 \leq \gamma L<\tau$, where $\tau=1-\sqrt{1-\mu\left(2 \eta-\mu k^{2}\right)}$. For an arbitrary $x_{1} \in C$, consider the sequence $\left\{x_{n}\right\}$ generated by the following iterative process:

$$
\begin{gathered}
x_{1} \in C, \\
x_{n+1}=P_{C}\left[\alpha_{n} \gamma V x_{n}+\left(I-\alpha_{n} \mu F\right) T x_{n}\right] \quad \forall n \in \mathbb{N},
\end{gathered}
$$

where $\left\{\alpha_{n}\right\}$ is a sequence in $(0,1)$ satisfying the conditions $(a)$ and $(b)$ of Theorem 3.1 .

Then, the sequence $\left\{x_{n}\right\}$ converges strongly to $\tilde{x} \in F(T)$, where $\tilde{x}$ is the unique solution of the following variational inequality:

$$
\langle(\mu F-\gamma V) \tilde{x}, \tilde{x}-y\rangle \leq 0 \quad \forall y \in F(T)
$$

Again, we derive the result of Tian ([11], Theorem 3.2) as the following corollary.

Corollary 3.3. Let $H$ be a real Hilbert space. Let $f$ be an $\alpha$-contraction on $H$ and $F: H \rightarrow H$ be a $k$-Lipschitzian and $\eta$-strongly monotone operator. Let $T: H \rightarrow H$ be a nonexpansive mapping such that $F(T) \neq \emptyset$. Suppose that $0<\mu<2 \eta / k^{2}$ and $0<\gamma \alpha<\tau$, where $\tau=\mu\left(\eta-\mu k^{2} / 2\right)$. For an arbitrary $x_{1} \in H$, consider the sequence $\left\{x_{n}\right\}$ generated by the following iterative process:

$$
\begin{gathered}
x_{1} \in H, \\
x_{n+1}=\alpha_{n} \gamma f\left(x_{n}\right)+\left(I-\alpha_{n} \mu F\right) T x_{n} \quad \forall n \in \mathbb{N},
\end{gathered}
$$

where $\left\{\alpha_{n}\right\}$ is a sequence in $(0,1)$ satisfying the conditions $(a)$ and $(b)$ of Theorem 3.1.

Then, the sequence $\left\{x_{n}\right\}$ converges strongly to $\tilde{x} \in F(T)$, where $\tilde{x}$ is the unique solution of the following variational inequality:

$$
\langle(\mu F-\gamma f) \tilde{x}, \tilde{x}-y\rangle \leq 0 \quad \forall y \in F(T)
$$

The following result obtains immediately from Theorem 3.1.

Corollary 3.4. Let $C$ be a nonempty closed convex subset of a real Hilbert space $H$. Let $F: C \rightarrow H$ be a $k$-Lipschitzian and $\eta$-strongly monotone operator and $V: C \rightarrow H$ be an L-Lipschitzian mapping. Let $\left\{T_{n}\right\}$ be a sequence of nonexpansive mappings from $C$ into itself such that $\bigcap_{n=1}^{\infty} F\left(T_{n}\right) \neq \emptyset$ and $T$ be a mapping from $C$ into itself defined by $T x=\lim _{n \rightarrow \infty} T_{n} x$ for all $x \in C$. Suppose that $0<\mu<2 \eta / k^{2}$ and $0 \leq \gamma L<\tau$, where $\tau=1-\sqrt{1-\mu\left(2 \eta-\mu k^{2}\right)}$. For an arbitrary $x_{1} \in C$, consider the sequence $\left\{x_{n}\right\}$ in $C$ generated by the following iterative process:

$$
\begin{gathered}
x_{1} \in C, \\
x_{n+1}=P_{C}\left[\alpha_{n} \gamma V x_{n}+\left(I-\alpha_{n} \mu F\right) T_{n} x_{n}\right] \quad \forall n \in \mathbb{N},
\end{gathered}
$$

where $\left\{\alpha_{n}\right\}$ is a sequence in $(0,1)$ satisfying the conditions (a)-(c) of Theorem 3.1. 
Then, the sequence $\left\{x_{n}\right\}$ converges strongly to $\tilde{x} \in \bigcap_{n=1}^{\infty} F\left(T_{n}\right)$, where $\tilde{x}$ is the unique solution of the following variational inequality:

$$
\langle(\mu F-\gamma V) \tilde{x}, \tilde{x}-y\rangle \leq 0 \quad \forall y \in \bigcap_{n=1}^{\infty} F\left(T_{n}\right) .
$$

\section{Application}

Recall that the so-called problem of image recovery is essentially to find a common element of finitely many nonexpansive retracts $C_{1}, C_{2}, \ldots, C_{r}$ of $C$ with $\bigcap_{i=1}^{r} C_{i} \neq \emptyset$. It is easy to see that every nonexpansive retraction $P_{i}$ of $C$ onto $C_{i}$ is a nonexpansive mapping of $C$ into itself. There is no doubt that the problem of image recovery is equivalent to finding a common fixed point of finitely many nonexpansive mappings $P_{1}, P_{2}, \ldots, P_{r}$ of $C$ into itself. Applying our main result, we obtain the following result which improves a number of results connected to the problem of image recovery.

Theorem 4.1. Let $C$ be a nonempty closed convex subset of a real Hilbert space $H$. Let $F: C \rightarrow$ $H$ be a $k$-Lipschitzian and $\eta$-strongly monotone operator and $V: C \rightarrow H$ be an L-Lipschitzian mapping. Let $\lambda_{i}>0(i=1,2,3, \ldots, N)$ such that $\sum_{i=1}^{N} \lambda_{i}=1$ and $T_{1}, T_{2}, T_{3}, \ldots, T_{N}: C \rightarrow C$ be nonexpansive mappings such that $\bigcap_{i=1}^{N} F\left(T_{i}\right) \neq \emptyset$. Suppose that $0<\mu<2 \eta / k^{2}$ and $0 \leq \gamma L<\tau$,

where $\tau=1-\sqrt{1-\mu\left(2 \eta-\mu k^{2}\right)}$. For an arbitrary $x_{1} \in C$, consider the sequence $\left\{x_{n}\right\}$ in $C$ generated by the following iterative process:

$$
\begin{gathered}
x_{1} \in C, \\
x_{n+1}=P_{C}\left[\alpha_{n} \gamma V x_{n}+\left(I-\alpha_{n} \mu F\right) \sum_{i=1}^{N} \lambda_{i} T_{i} x_{n}\right] \quad \forall n \in \mathbb{N},
\end{gathered}
$$

where $\left\{\alpha_{n}\right\}$ is a sequence in $(0,1)$ satisfying the conditions $(a)$ and $(b)$ of Theorem 3.1.

Then, the sequence $\left\{x_{n}\right\}$ converges strongly to $\tilde{x} \in \bigcap_{i=1}^{N} F\left(T_{i}\right)$, where $\tilde{x}$ is the unique solution of the following variational inequality:

$$
\langle(\mu F-\gamma V) \tilde{x}, \tilde{x}-y\rangle \leq 0 \quad \forall y \in \bigcap_{i=1}^{N} F\left(T_{i}\right) .
$$

Proof. Define $T=\sum_{i=1}^{N} \lambda_{i} T_{i}$. Then $T$ is nonexpansive mapping from $C$ into itself. Thus, using Lemma 2.6, we get $F(T)=\bigcap_{i=1}^{N} F\left(T_{i}\right)$. Therefore, the proof follows from Corollary 3.2.

\section{Numerical Example}

For showing the effectiveness and convergence of the sequence generated by the considered iterative scheme, we discuss the following example.

Example 5.1. Let $H=\mathbb{R}$ and $C=[0,1]$. Let $T$ be a self-mapping defined by $T x=1-x$ for all $x \in C$. Let $F, V: C \rightarrow H$ be two mappings defined by $F x=4 x$ and $V x=2 x$ for all $x \in C$, 


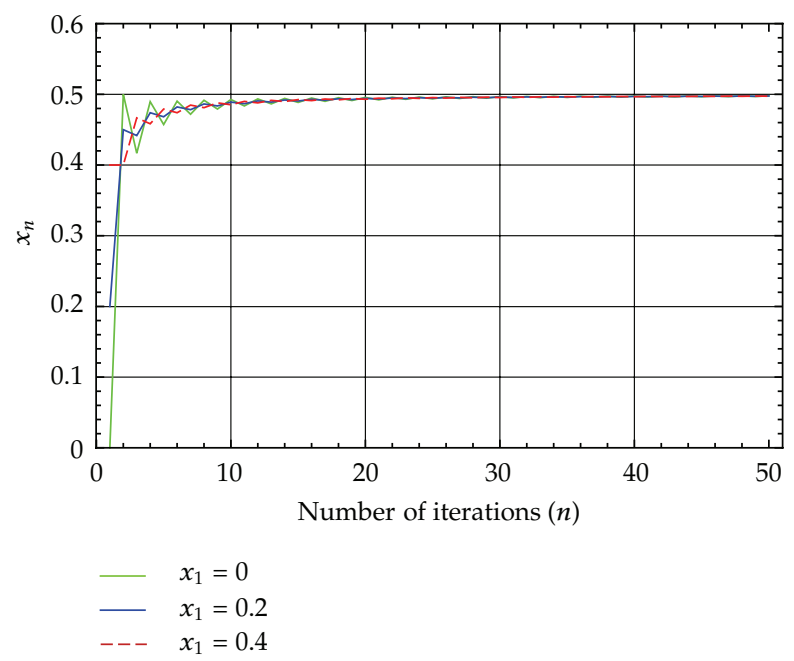

Figure 1

where $F$ is a $k$-Lipschitzian and $\eta$-strongly monotone, and $V$ is an $L$-Lipschitzian mapping. We take $0<\mu<2 \eta / k^{2}$ and $0 \leq \gamma L<\tau$, and we have $\mu=1 / 4, \tau=1$ and $\gamma=1 / 4$. Define $\left\{\alpha_{n}\right\}$ in $(0,1)$ by $\alpha_{n}=1 / n+1$. Without loss of generality, we may assume that $a_{n}=1 / n^{3 / 2}$ for all $n \in \mathbb{N}$. For each $n \in \mathbb{N}$, define $T_{n}: C \rightarrow C$ by

$$
T_{n} x= \begin{cases}1-x, & \text { if } x \in[0,1) \\ a_{n}, & \text { if } x=1\end{cases}
$$

In [15], it is proved that $\tau=\left\{T_{n}\right\}$ is a sequence of nearly nonexpansive mappings from $C$ into itself such that $F(\tau)=\{1 / 2\}$ and $T x=\lim _{n \rightarrow \infty} T_{n} x$ for all $x \in C$, where $T$ is nonexpansive mapping.

It can be observed that all the assumptions of Theorem 3.1 are satisfied and the sequence $\left\{x_{n}\right\}$ generated by (3.1) converges to a unique solution $1 / 2$ of variational inequality (3.2) over $F(\tau)$. The graphical presentation of the convergence of the sequence $\left\{x_{n}\right\}$ generated by the iterative scheme (3.1) is given in Figure 1.

\section{Acknowledgments}

The authors would like to thank the referees for useful comments and suggestions.

\section{References}

[1] R. P. Agarwal, D. O'Regan, and D. R. Sahu, Fixed Point Theory for Lipschitzian-Type Mappings with Applications, Topological Fixed Point Theory and Its Applications, Springer, New York, NY, USA, 2009.

[2] D. R. Sahu, "Fixed points of demicontinuous nearly Lipschitzian mappings in Banach spaces," Commentationes Mathematicae Universitatis Carolinae, vol. 46, no. 4, pp. 653-666, 2005.

[3] A. Moudafi, "Viscosity approximation methods for fixed-points problems," Journal of Mathematical Analysis and Applications, vol. 241, no. 1, pp. 46-55, 2000. 
[4] H. K. Xu, "Viscosity approximation methods for nonexpansive mappings," Journal of Mathematical Analysis and Applications, vol. 298, no. 1, pp. 279-291, 2004.

[5] G. Marino and H. K. Xu, "A general iterative method for nonexpansive mappings in Hilbert spaces," Journal of Mathematical Analysis and Applications, vol. 318, no. 1, pp. 43-52, 2006.

[6] Y. Liu, "A general iterative method for equilibrium problems and strict pseudo-contractions in Hilbert spaces," Nonlinear Analysis, Theory, Methods and Applications, vol. 71, no. 10, pp. 4852-4861, 2009.

[7] X. Qin, M. Shang, and S. M. Kang, "Strong convergence theorems of modified Mann iterative process for strict pseudo-contractions in Hilbert spaces," Nonlinear Analysis, Theory, Methods and Applications, vol. 70, no. 3, pp. 1257-1264, 2009.

[8] S. Wang, "Convergence and weaker control conditions for hybrid iterative algorithms," Fixed Point Theory and Applications, vol. 2011, no. 1, article 3, 14 pages, 2011.

[9] S. Wang, "Two general algorithms for computing fixed points of nonexpansive mappings in Banach spaces," Journal of Applied Mathematics, vol. 2012, Article ID 658905, 11 pages, 2012.

[10] S. Wang and C. Hu, "Two new iterative methods for a countable family of nonexpansive mappings in Hilbert spaces," Fixed Point Theory and Applications, vol. 2010, Article ID 852030, 12 pages, 2010.

[11] M. Tian, "A general iterative algorithm for nonexpansive mappings in Hilbert spaces," Nonlinear Analysis, Theory, Methods and Applications, vol. 73, no. 3, pp. 689-694, 2010.

[12] I. Yamada, "The hybrid steepest descent method for the variational inequality problem over the intersection of fixed point sets of nonexpansive mappings," in Inherently Parallel Algorithms in Feasibility and Optimization and Their Applications (Haifa, 2000), D. Butnariu, Y. Censor, and S. Reich, Eds., vol. 8, pp. 473-504, Studies in Computational Mathematics, Amsterdam, The Netherlands, 2001.

[13] L. C. Ceng, Q. H. Ansari, and J. C. Yao, "Some iterative methods for finding fixed points and for solving constrained convex minimization problems," Nonlinear Analysis, Theory, Methods and Applications, vol. 74, no. 16, pp. 5286-5302, 2011.

[14] K. Aoyama, Y. Kimura, W. Takahashi, and M. Toyoda, “Approximation of common fixed points of a countable family of nonexpansive mappings in a Banach space," Nonlinear Analysis, Theory, Methods and Applications, vol. 67, no. 8, pp. 2350-2360, 2007.

[15] N. C. Wong, D. R. Sahu, and J. C. Yao, "A generalized hybrid steepest-descent method for variational inequalities in Banach spaces," Fixed Point Theory and Applications, vol. 2011, Article ID 754702, 28 pages, 2011.

[16] K. Goebel and W. A. Kirk, Topics on Metric Fixed Point Theory, Cambridge University Press, Cambridge, UK, 1990.

[17] H. K. Xu and T. H. Kim, "Convergence of hybrid steepest-descent methods for variational inequalities," Journal of Optimization Theory and Applications, vol. 119, no. 1, pp. 185-201, 2003.

[18] N. C. Wong, D. R. Sahu, and J. C. Yao, "Solving variational inequalities involving nonexpansive type mappings," Nonlinear Analysis, Theory, Methods and Applications, vol. 69, no. 12, pp. 4732-4753, 2008. 


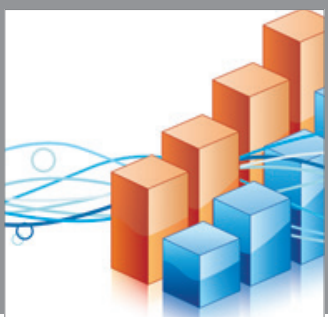

Advances in

Operations Research

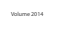

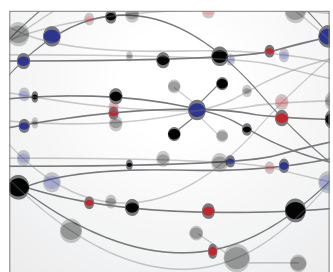

\section{The Scientific} World Journal
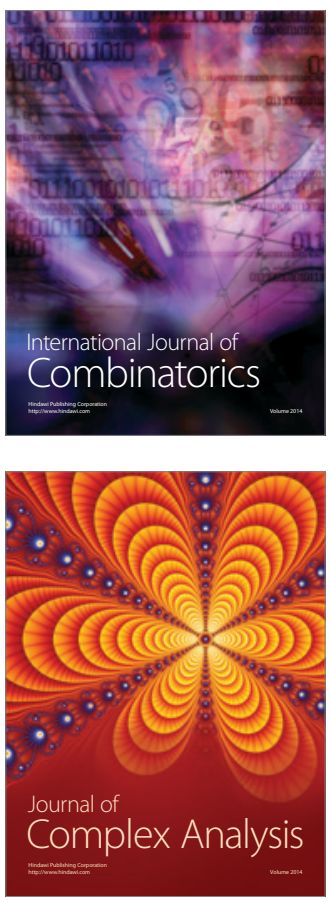

International Journal of

Mathematics and

Mathematical

Sciences
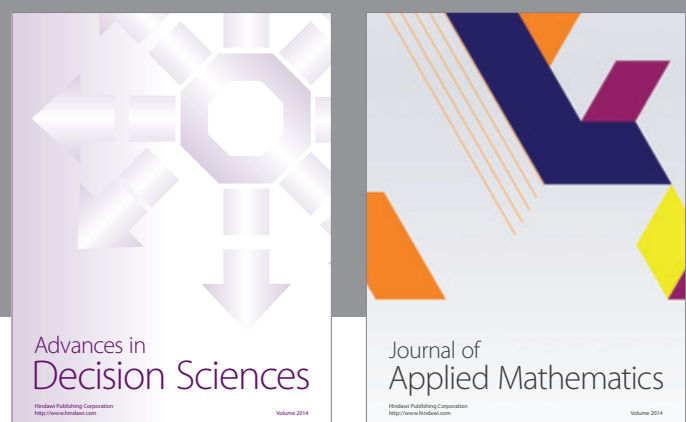

Journal of

Applied Mathematics
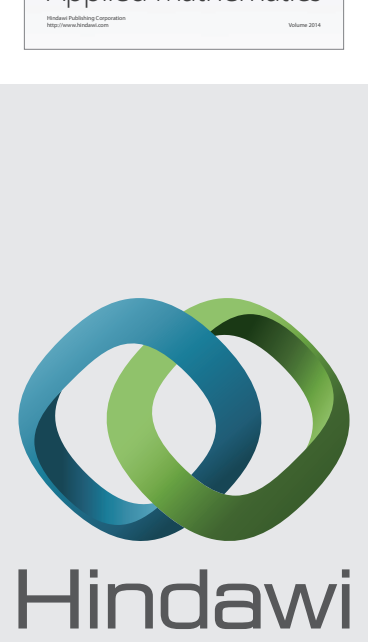

Submit your manuscripts at http://www.hindawi.com
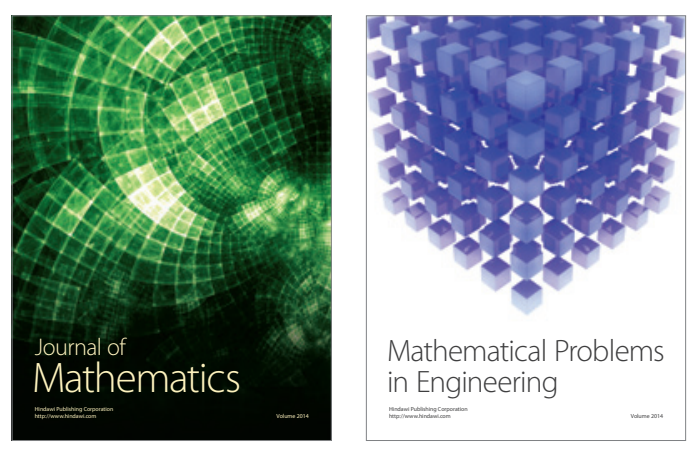

Mathematical Problems in Engineering
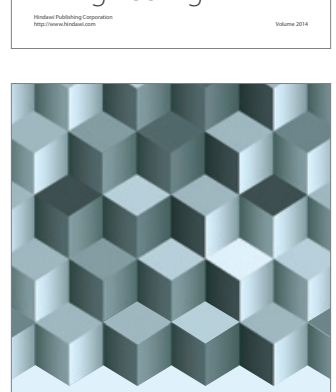

Journal of

Function Spaces
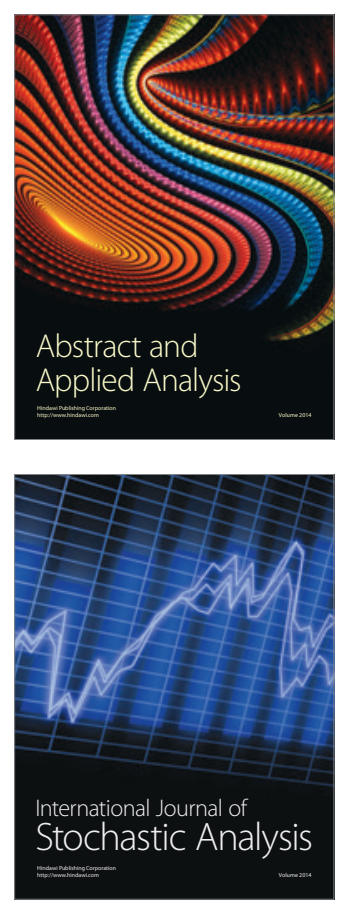

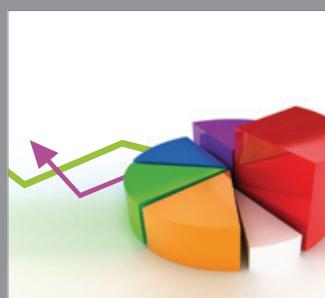

ournal of

Probability and Statistics

Promensencen
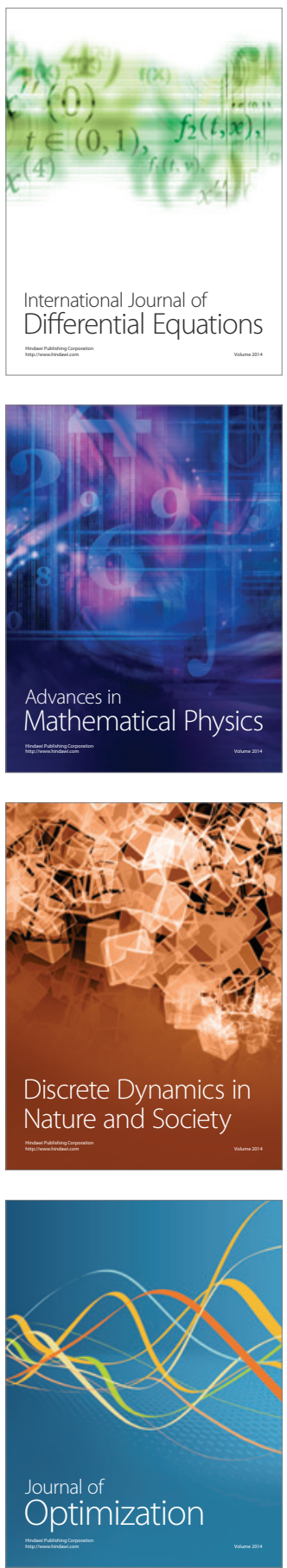